\title{
A Belief Rule Based Expert System for Evaluating Technological Innovation Capability of High-Tech Firms Under Uncertainty
}

\author{
Mohammad Newaj Jamil \\ Department of Computer \\ Science and \\ Engineering \\ University of Chittagong \\ Chittagong, Bangladesh \\ hridoyjamil10@gmail.com
}

\author{
Mohammad Shahadat Hossain \\ Department of Computer \\ Science and \\ Engineering \\ University of Chittagong \\ Chittagong, Bangladesh \\ hossain_ms@cu.ac.bd
}

\author{
Raihan Ul Islam \\ Department of Computer \\ Science, Electrical \\ and Space Engineering \\ Luleå University of \\ Technology \\ Skellefteå, Sweden \\ raihan.ul.islam@ltu.se
}

\author{
Karl Andersson \\ Department of Computer \\ Science, Electrical \\ and Space Engineering \\ Luleå University of \\ Technology \\ Skellefteå, Sweden \\ karl.andersson@ltu.se
}

\begin{abstract}
Technological innovation capability (TIC) is a complicated and subtle concept which is based on multiple quantitative and qualitative criteria. The cores of a firm's long-term competitive dominance are defined by technological innovation capability which is the incentive for a firm's innovation. Various types of uncertainty can be noticed while considering multiple criteria for evaluating TIC. In order to evaluate TIC in a reliable way, a Belief Rule Base (BRB) Expert System can be used to handle both quantitative and qualitative data and their associated uncertainties. In this paper, a RESTful API-based BRB expert system is introduced to evaluate technological innovation capability by taking uncertainties into consideration. This expert system will facilitate firms' managers to obtain a recapitulation of the TIC evaluation. It will help them to take essential steps to ensure corporate survival and strengthen their weak capabilities continuously to facilitate a competitive advantage. Other users can also use this API to apply BRB for a different domain. However, a comparison between the knowledge-driven approach (BRBES) and several data-driven models has been performed to find out the reliability in evaluating TIC. The result shows that the outcome of BRBES is better than other data-driven approaches.
\end{abstract}

Keywords-Technological Innovation Capability, Belief Rule Base, Uncertainty, RESTful API.

\section{INTRODUCTION}

Nowadays firms are facing rapidly changing environments, so they require continuous technological innovation and managerial response for maintaining competitiveness. A firm needs to reorganize its organizational assets and enhance its competitiveness to attain a satisfactory degree of harmonization to the external environment. Product life cycles shortening and globalization of markets has accelerated firm response rapidly to improve and refine their technology management activities [1]. Hence identification and evaluation of technologies from numerous sources can play a significant role in the efficient management of technological resources. For this reason, firms must combine organizational resources, and technological innovation so that they can assure corporate survival and competitive advantage. Technological innovation capabilities have become the principal foundation of market competition.
Firms need to monitor their TICs periodically and strengthen their weak capabilities continuously to facilitate a competitive advantage. Technological innovation capabilities consist of multi-criteria difficulties and involve various organizational tasks and resource combination among several departments [2]. These capabilities are important for the firms' continuous improvement in a competitive and sustainable market. A firm's technological innovation-related activities contain high uncertainty and imprecision. Therefore, it is difficult to assess innovation processes correctly as they are uncertain and unpredictable [3]. Technological innovation contains three types of uncertainties, which are the technological, market and enterprise-based uncertainties [4]. From this viewpoint, each phase of the technological innovation process embeds numerous sources of uncertainty and ambiguities. Since a firm's technological innovation capabilities are typically subjective and imprecise, this subjectivity and imprecision make the TICs evaluation process more complicated and challenging. Thus, evaluators may have different perspectives regarding various criteria and objectives. Technological innovation capabilities of a firm contain numerous criteria. These criteria can be both quantitative and qualitative criteria. Thus, several types of uncertainty exist while measuring these criteria which can be vagueness, imprecision, ambiguity, ignorance, and incompleteness. Insufficient data or lack of human knowledge can be one of the reasons for causing these uncertainties. For addressing this issue, a Belief Rule-Based Expert System (BRBES) can be utilized which can manage both quantitative and qualitative data and their related uncertainties to evaluate technological innovation capabilities efficiently. BRB expert system uses belief rule base for representing uncertain knowledge, and Evidential Reasoning (ER) works as an inference engine to manage both uncertain and heterogeneous data [5]. In this paper, a RESTful API-Based BRB Expert System has been developed which can process heterogeneous data with various types of uncertainties for evaluating TICs. Establishing a RESTful API-Based BRB expert system facilitates the extensive use of the system for different domains. For this research, a performance evalua- 
tion system like the evaluation of Technological Innovation Capability (TIC) is taken as a use case. The remainder of this article is structured as follows: Section II covers related work on technological innovation capability evaluation, while Section III provides an overview of methodology. Section IV describes the RESTful API-based BRB Expert System, and Section V presents the results and discussion. Finally, Section VI concludes the paper and indicates future work.

\section{RELATED WORK}

There are several existing methods used previously for evaluating TIC. Yam et al. [6] used statistical regression analysis to determine the TICs of Chinese firms in Beijing, China, based on seven capabilities which are learning, $R \& D$, resource allocation, manufacturing, marketing, organizing and strategic planning. The relationship between TICs and innovation rate, product competitiveness, and sales growth among the firms was determined by utilizing regression analysis. Lu et al. [7] used the Analytical Hierarchy Process (AHP), fuzzy approach and multi-criteria together, where evaluators subjective judgments were used to make the TICs decision structure explicit based on their decision structure. An Analytical Hierarchy Process (AHP) method was used to determine the weight of all aspects and criteria of innovation performance, while evaluators' subjective judgments were made by applying the fuzzy set theory, and the fuzzy Multiple Attribute DecisionMaking (MADM) method was employed to evaluate the firm innovation performance. Wang et al. [2] used a fuzzy measure and non-additive fuzzy integral method to assess the performance of synthetic technological innovation capabilities in hitech firms. The non-additive fuzzy integral method was employed to identify the principal criteria impacting TICs at hitech firms. Kong et al. [8] evaluated technological innovation capability by applying a fuzzy decision-making approach. The weight of subjective judgments was determined by employing an Analytic Network Process (ANP), while the fuzzy VIKOR algorithm was used to derive the best technology innovation enterprise. Cheng et al. [9] employed trapezoid fuzzy numbers and extension of Technique for Order Performance by Similarity to Ideal Solution (TOPSIS) for addressing the evaluation of technological innovation capabilities. This hybrid method was used to assess a printed circuit board firm. Sumrit et al. [10] employed the Decision Making Trial and Evaluation Laboratory (DEMATEL) method for examining the significance of criteria and establishing the causal connections among the criteria to evaluate the TICs of enterprises. All these TIC evaluation methods address various issues of technological innovation capabilities for specific scenarios. However, linear regression and DEMATEL method failed to address uncertainty in data as they don't have any mechanism to address uncertainty. Although fuzzy based approaches can handle uncertainties as a result of imprecision, vagueness, and ambiguity, they cannot address uncertainties because of incompleteness and ignorance, which can be observed with the criteria for evaluating technological innovation capability. Therefore, an appropriate knowledge representation schema and reasoning mechanism need to be used to address different kinds of uncertainties existing with the criteria of TICs. So, Belief Rule Base (BRB) can be utilized which will be an efficient way to evaluate technological innovation capability.

\section{Methodology}

A Belief Rule-Based Expert System (BRBES) consists of two main components which are a knowledge base and an inference engine. BRB expert system uses Belief Rule Base (BRB) for representing uncertain knowledge and creating the initial knowledge base, while Evidential Reasoning (ER) works as an inference engine to handle heterogeneous and uncertain data. [5]. A Belief Rule Base (BRB) is an extended form of conventional IF-THEN rule base, and it can express more complex non-linear causal connections under uncertainty. There are two main parts in a belief rule which are antecedent and consequent. Each antecedent attribute is linked with referential values while belief degrees are embedded with consequent attributes. BRB contains various learning or knowledge representation parameters such as attribute weight, rule weight, and belief degrees which are used to capture uncertainty in data [11], [12], [13]. A belief rule is presented below:

IF Fund Raising Ability is High AND Optimal Capital Allocation is High AND Intensity of Capital Input is Medium AND Return on Investment is Low THEN Capital Capabilities is (High, 0.3), (Medium, 0.7), (Low, 0.0)

In this rule, "Fund Raising Ability", "Optimal Capital Allocation", "Intensity of Capital Input" and "Return on Investment" are the antecedent attributes. "High", "Medium" and "Low" are their corresponding referential values. "Capital Capabilities" is the consequent attribute and it's referential values are "High", "Medium" and "Low". The belief distribution of the consequent "Capital Capabilities" are (High, 0.3), (Medium, 0.7 ) and (Low, 0$)$. The sum of belief degrees $(0.3+0.7+0.0$ $=1$ ) associated with each referential values of the consequent attribute is one, so this rule can be said complete. If the sum of belief degrees is less than one, then the rule can be considered as incomplete. It can cause because of ignorance or incompleteness. The relationship between antecedents and the consequent attribute is linear in traditional IF-THEN rule while it is non-linear for belief rule. In addition, data obtained from interviews or surveys are naturally non-linear [13]. As a result, it is likely to use belief rules for representing the data effectively. Evidential Reasoning (ER) can handle heterogeneous data as well as different types of uncertainties, for instance, incompleteness, ignorance, imprecision, vagueness [14], [15]. The inference procedures using the ER approach consists of various steps such as input transformation, rule activation weight calculation, belief update, and rule aggregation which is shown in Fig. 1. When input transformation is performed, the input value of an antecedent attribute is distributed over the referential values of that antecedent attribute of a rule, and these transformed values of the input data are known as matching degrees. The rules are called packet antecedent after assigning the matching degree, and they become active. 


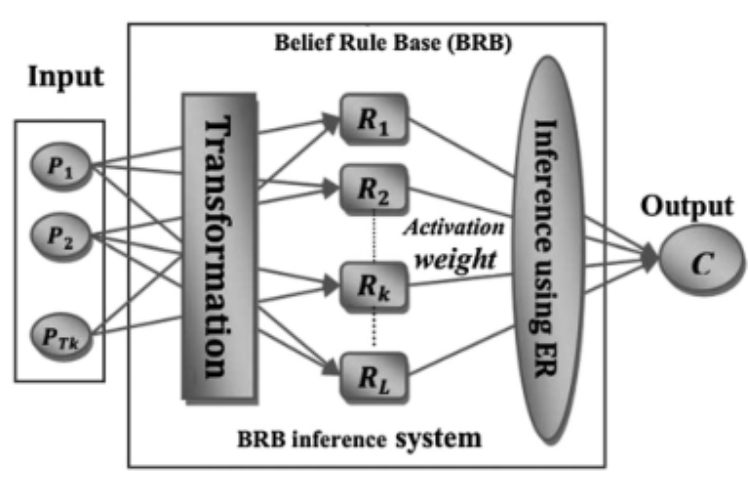

Fig. 1. Sequence of BRBES Inference Procedures

The individual matching degrees of the antecedent attributes of a rule are combined using a weighted multiplicative equation. This combined matching degree of a rule is utilized to calculate the activation weight of that rule. The activation weight of a rule will be zero if that rule is not activated. After calculating the sum of the rule activation weight of a rule base, the result should be one [16], [17]. If there is an absence of data for any antecedent attributes of a rule base because of ignorance, the initial belief degrees embedded with each rule in the rule base need to be updated to address the uncertainty due to ignorance. Finally, all the packet antecedents of the rules are aggregated to calculate the output for the input data of the antecedent attributes using the Evidential Reasoning algorithm. The calculated output value against the input data will be in a fuzzy form. The utility score embedded with each referential value of the consequent attribute is utilized to convert this fuzzy value into a crisp or numerical value. All these steps are done by following the procedures mentioned in [18], [19].

\section{BRBES to EVAluate TEChNOLOGICAL INNOVATION CAPABILITY}

This section describes the architecture of the RESTful APIbased BRB expert system for evaluating technological innovation capability. Previously, a RESTful API was developed for flood risk assessment [20]. But there was a major flaw in the tree traversal algorithm when creating BRB dynamically. In the last iteration, it includes the topmost node as antecedent as well as consequent. In this paper, this flaw has been fixed by developing a new tree traversal algorithm. Besides, the previous RESTful API does not work if the referential value of antecedent and consequent is more than three. The new RESTful API will work even if the referential value of antecedent and consequent is more than three. However, in previous RESTful API, eleven keys were needed for a node in JSON data. In the new RESTful API, we only need eight keys for a node in JSON data. So, it will be less time consuming to prepare JSON data. Moreover, there is some limitation in previous RESTful API. The output shown for the initial rule base was only for the top-level BRB. For multi-level BRB, the initial rule base for mid-level BRB was not shown as output. As a result, the user gets no idea regarding the midlevel BRB. In addition, the output didn't show the antecedent and consequent of the corresponding rule. In the new RESTful API, the output of the initial rule base is shown for the toplevel BRB as well as mid-level BRB, and the output shows the antecedent and consequent of corresponding rules for all BRB. Furthermore, in previous RESTful API, the output shown for transformed input, activation weight, belief update, and rule aggregation was only for top-level BRB. In the new RESTful API, the output for all these steps is for top-level BRB as well as the mid-level BRB.

\section{A. Architecture of RESTful API}

The RESTful API follows a three-layer architecture model which are data management layer, application layer, and Application Programming Interface (API) layer. The architecture of RESTful API is represented in Fig. 2.

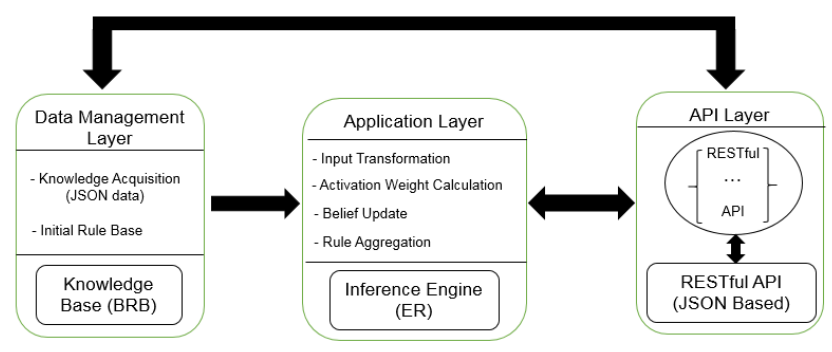

Fig. 2. Architecture of RESTful API

1) Data Management Layer: Data management layer is accountable to create the initial rule base using the data. The initial BRB is the knowledge base of the system which is created in the data management layer. A BRB framework is created manually to construct the knowledge base while taking the criteria associated with the evaluation of technological innovation capability, which is shown in Fig. 3. In this framework, five aspects and their associated criteria have been considered for TIC evaluation. These aspects and criteria are defined in [2]. In this research, JSON is used as the data format. The data for the node 'Percentage of researchers' is shown in JSON format in Fig. 4.

2) Application Layer: Application layer contains inference engine with procedures such as input transformation, rule activation weight calculation, belief update, and rule aggregation. The input of the application layer is the initial BRB from the data management layer, and it runs the inference procedure on it. The initial BRB tree can be multi-level, so we need a tree traversal algorithm to traverse the BRB tree dynamically. Here, the previous tree traversal algorithm [20] has been modified, and a new dynamic tree traversal algorithm has been developed to fix the flaw as mentioned earlier. It uses a bottom-up approach to perform BRB tree traversal like the previous algorithm. This algorithm has also linear time and space complexity. The algorithm is demonstrated in Algorithm 1. The whole BRB tree can be traversed from bottom to top by 


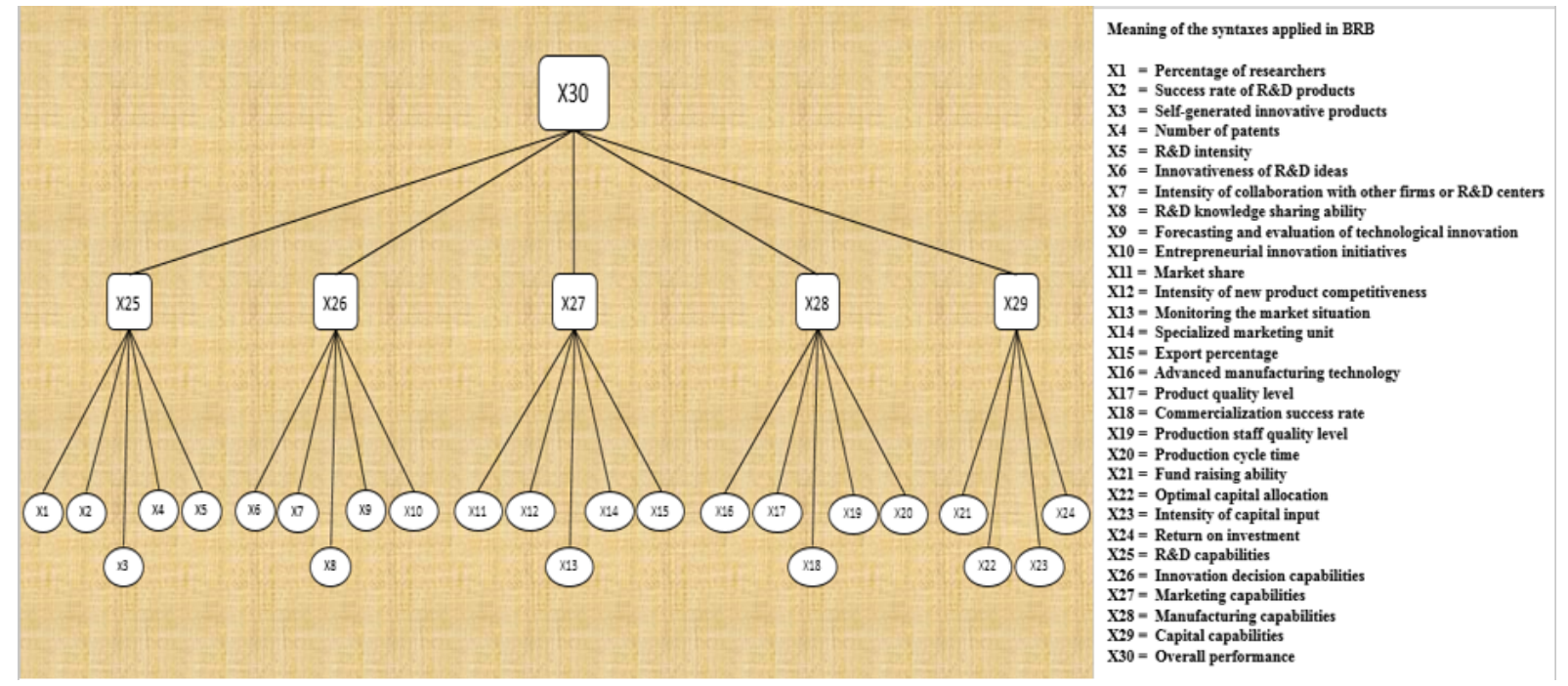

Fig. 3. BRB Framework to Evaluate Technological Innovation Capability

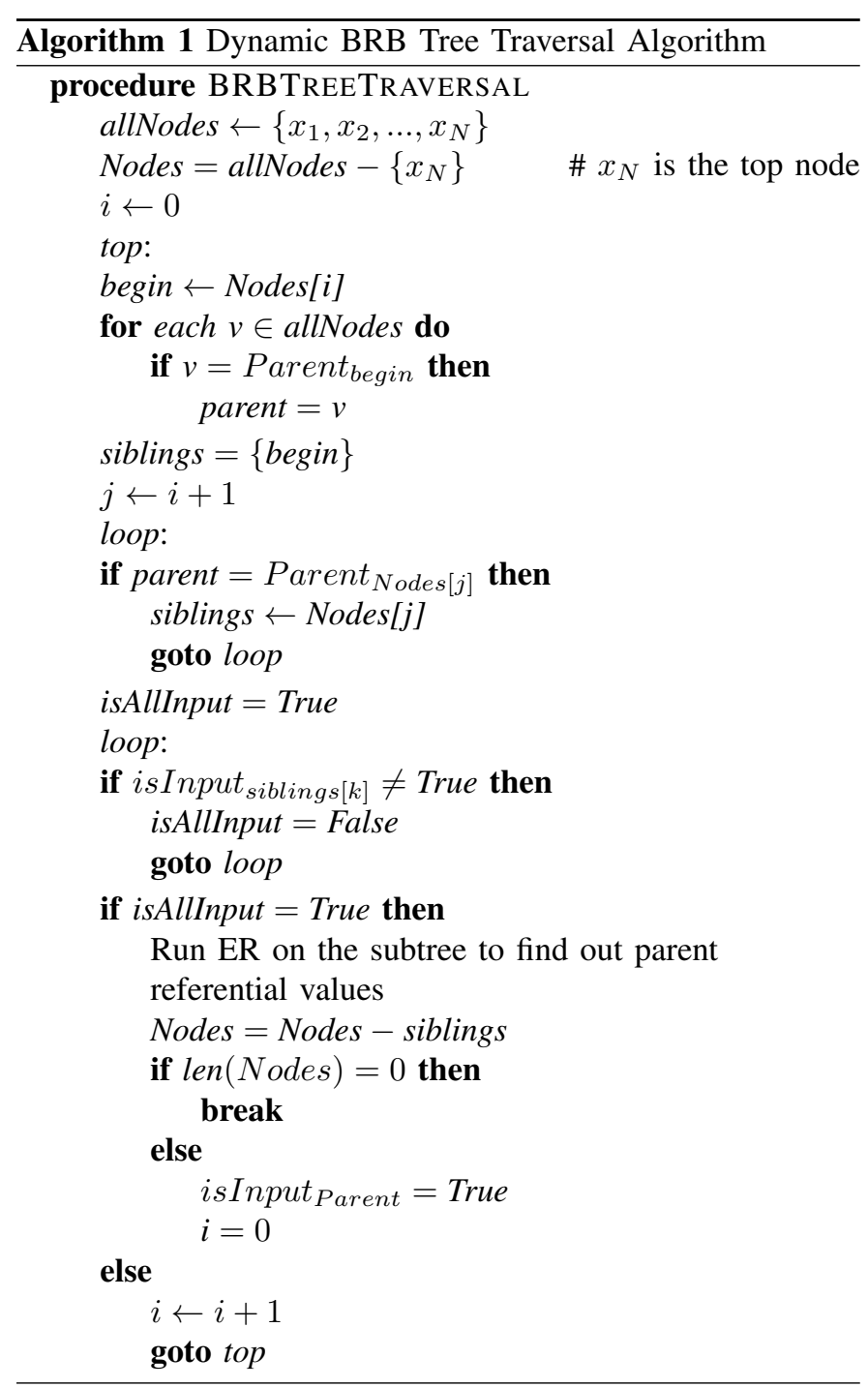

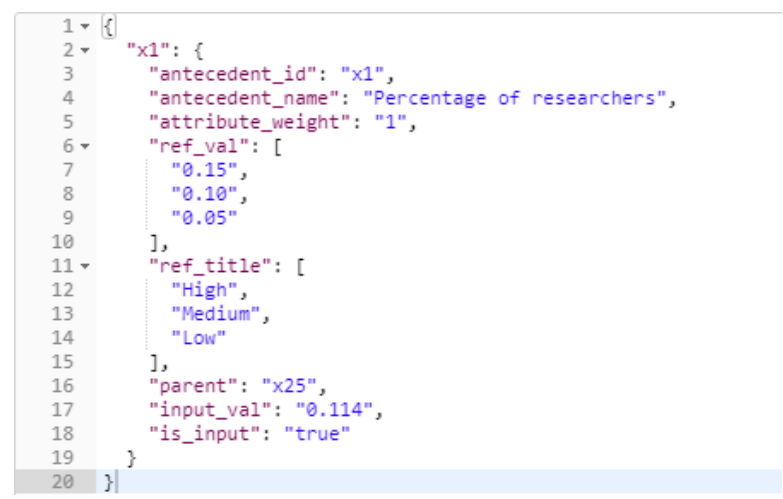

Fig. 4. JSON data format for a node in BRB framework

using this algorithm, and the result of the top node is generated by calculating the subtrees gradually.

3) API Layer: API Layer provides a simple programming interface to produce the output of different levels of the BRBES. This API facilitates a communication channel for the users using a high level of abstraction by contacting with the data management and application layer. It communicates with HTTP verbs and uses Uniform Resource Locator (URL), which is also known as API endpoints. The procedure to use this API is the same as mentioned in [20].

\section{REsults And Discussion}

In order to evaluate technological innovation capability, data are collected from the multi-source database to increase accuracy and decrease error. Since many high-tech firms have no complete coverage of standard financial statistics and the data is treated as secret, it is troublesome to gather all the quantitative data from each high-tech firm. Therefore, the quantitative data are collected from each high-tech firm's annual operation report from various sources. The qualitative data are collected from different surveys where the senior 
TABLE I

DIFFERENT CRITERIA OF TEN FIRMS FOR TIC EVALUATION

\begin{tabular}{|c|c|c|c|c|c|c|c|c|c|c|c|c|c|c|c|c|c|c|c|c|c|c|c|c|}
\hline Firm No. & $x_{1}$ & $x_{2}$ & $x_{3}$ & $x_{4}$ & $x_{5}$ & $x_{6}$ & $x_{7}$ & $x_{8}$ & $x_{9}$ & $x_{10}$ & $x_{11}$ & $x_{12}$ & $x_{13}$ & $x_{14}$ & $x_{15}$ & $x_{16}$ & $x_{17}$ & $x_{18}$ & $x_{19}$ & $x_{20}$ & $x_{21}$ & $x_{22}$ & $x_{23}$ & $x_{24}$ \\
\hline 1 & 0.087 & 0.467 & 10 & 201 & 565.220 & 0.66 & 1.00 & 1.00 & 0.66 & 1.00 & 0.174 & 0.33 & 1.00 & 1.00 & 0.858 & 0.66 & 0.33 & 0.309 & 1.00 & 10 & 0.33 & 1.00 & 1.00 & 0.161 \\
\hline 2 & 0.081 & 0.625 & 30 & 290 & 543.174 & 0.66 & 1.00 & 0.66 & 0.33 & 0.66 & 0.201 & 0.33 & 0.66 & 1.00 & 0.800 & 0.66 & 0.66 & 0.418 & 0.66 & 14 & 1.00 & 0.66 & 0.33 & 0.133 \\
\hline 3 & 0.053 & 0.461 & 22 & 314 & 418.772 & 1.00 & 0.33 & 0.33 & 0.33 & 1.00 & 0.057 & 1.00 & 0.66 & 0.66 & 0.654 & 0.33 & 0.33 & 0.475 & 0.33 & 11 & 0.66 & 1.00 & 1.00 & 0.217 \\
\hline 4 & 0.086 & 0.384 & 11 & 312 & 300.819 & 0.33 & 0.66 & 1.00 & 1.00 & 1.00 & 0.157 & 1.00 & 1.00 & 1.00 & 0.823 & 1.00 & 0.33 & 0.519 & 1.00 & 10 & 0.66 & 0.33 & 0.66 & 0.313 \\
\hline 5 & 0.104 & 0.369 & 16 & 248 & 734.514 & 1.00 & 0.33 & 0.66 & 0.33 & 1.00 & 0.139 & 0.33 & 1.00 & 1.00 & 0.548 & 1.00 & 1.00 & 0.366 & 0.66 & 14 & 1.00 & 0.33 & 1.00 & 0.254 \\
\hline 6 & 0.104 & 0.602 & 12 & 327 & 801.184 & 1.00 & 0.33 & 1.00 & 0.33 & 1.00 & 0.188 & 0.33 & 0.33 & 0.66 & 0.910 & 1.00 & 1.00 & 0.518 & 1.00 & 12 & 0.66 & 0.66 & 0.33 & 0.205 \\
\hline 7 & 0.069 & 0.632 & 24 & 285 & 614.565 & 0.33 & 0.66 & 0.33 & 0.33 & 1.00 & 0.225 & 1.00 & 0.66 & 0.66 & 0.733 & 0.66 & 1.00 & 0.341 & 1.00 & 11 & 1.00 & 0.66 & 0.66 & 0.150 \\
\hline 8 & 0.074 & 0.376 & 22 & 320 & 661.094 & 0.33 & 0.66 & 0.66 & 1.00 & 1.00 & 0.038 & 0.33 & 0.66 & 1.00 & 0.466 & 1.00 & 0.33 & 0.290 & 1.00 & 10 & 0.33 & 0.33 & 0.33 & 0.235 \\
\hline 9 & 0.070 & 0.668 & 9 & 277 & 666.716 & 0.66 & 0.66 & 0.66 & 0.33 & 1.00 & 0.093 & 0.33 & 1.00 & 0.33 & 0.741 & 0.66 & 0.66 & 0.568 & 0.33 & 13 & 0.33 & 0.66 & 1.00 & 0.280 \\
\hline 10 & 0.080 & 0.567 & 21 & 317 & 504.636 & 0.66 & 0.66 & 1.00 & 1.00 & 0.33 & 0.092 & 0.33 & 0.33 & 0.33 & 0.557 & 1.00 & 1.00 & 0.378 & 1.00 & 10 & 0.33 & 1.00 & 0.66 & 0.193 \\
\hline
\end{tabular}

TABLE II

TECHNOLOGICAL INNOVATION CAPABILITY EVALUATION FOR TEN FIRMS BY BRBES, LINEAR REGRESSION, ANN, RANDOM FOREST AND DECISION TREE

\begin{tabular}{|c|c|c|c|c|}
\hline \multicolumn{5}{|c|}{$x_{30}$} \\
\hline BRBES & Linear Regression & ANN & Random Forest & Decision Tree \\
\hline 73.557 & 73.322 & 67.593 & 70.308 & 69.242 \\
\hline 71.903 & 71.8 & 69.209 & 64.493 & 67.372 \\
\hline 68.327 & 68.014 & 64.741 & 68.034 & 65.86 \\
\hline 69.684 & 70.212 & 69.524 & 68.218 & 61.696 \\
\hline 78.7 & 77.789 & 74.895 & 75.057 & 70.806 \\
\hline 70.882 & 71.591 & 68.432 & 65.413 & 63.655 \\
\hline 68.204 & 69.925 & 67.169 & 65.745 & 67.459 \\
\hline 61.407 & 62.074 & 62.231 & 58.886 & 57.347 \\
\hline 69.372 & 69.891 & 69.403 & 70.304 & 69.278 \\
\hline 60.562 & 60.699 & 62.063 & 63.445 & 59.067 \\
\hline
\end{tabular}

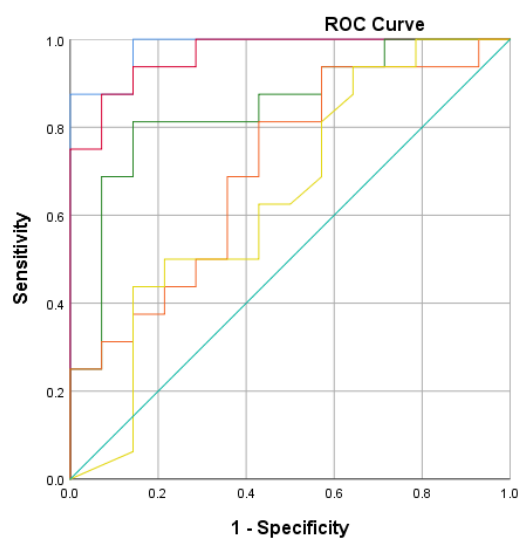

$\begin{gathered}\text { Source of the } \\ \text { Curve }\end{gathered}$
- BRBES
- LinearRegression
- ANN
- RandomForest
DecisonTree
- Reference Line

Fig. 5. Comparison of Results of BRBES, Linear Regression, ANN, Random Forest and Decision Tree Using ROC Curves

managers of each high-tech firms were asked to respond for evaluating current technological innovation performance level. Around 100 firms' data are collected from various sources, which can be considered as good enough because sample sizes in between 30 and 500 data points are considered appropriate for most research [21]. Based on the data, the TICs of each high-tech firms is evaluated using BRBES. A



Fig. 6. Comparison of AUC of BRBES, Linear Regression, ANN, Random Forest and Decision Tree

study with performance evaluation has been conducted to determine how BRBES, which is a knowledge-driven approach is performing compared to Linear Regression, ANN, Random Forest, and Decision Tree, which are data-driven models. Rules and logic are used by the knowledge-driven approach for representing beliefs. On the contrary, the data-driven approach does not use logic and only concentrates on observed data. Since data-driven approaches require data collected from past experiences, this makes them vulnerable in application to new things whether the knowledge-driven approach is not biased by the past and can employ new knowledge immediately. So, these two approaches can be competitive or cooperative when both observed data and expert knowledge of a specific domain is available. The machine learning methods were tested in the Spyder IDE. Thirty firms' data have been considered to perform a comparison between BRBES and the mentioned machine learning techniques. For simplicity, Table I presents different criteria of ten firms' for technological innovation capability evaluation and Table II presents BRBES and the mentioned machine learning techniques produced results. The reliability of one technique over other techniques can be achieved by comparing, evaluating, and assessing the accuracy of their results. Receiver Operating Characteristic (ROC) curves provide a comprehensive and perceptible representation of comparison, evaluation, and assessment of different techniques. Thus it is extensively used in different domains including clinical applications, atmospheric science, and many others. Therefore, ROC curves have been considered in this research to assess the accuracy and the reliability of the BRBES in comparison to other machine learning approaches. Area under curve (AUC) of ROC is utilized to measure the 
accuracy of a result. The greater value of AUC indicates more accuracy and reliability of the predictive models. Fig. 5 shows ROC curves for BRBES, Linear Regression, ANN, Random Forest and Decision Tree, while Fig. 6 shows the AUC and the confidence interval (CI) for the mentioned techniques. From Fig. 6, it can be noticed that the highest value of AUC is for BRBES because it considers all types of uncertainty that exist with both the qualitative and quantitative data which are required to evaluate TIC. However, this is not the case with the machine learning techniques as they do not consider uncertainties in the data. Due to the linear nature of the collected data, Linear Regression performs better than other machine learning techniques. That's why the AUC of Linear Regression is very near to BRBES. On the other hand, most of the time ANN doesn't deal well with small data. Though Random Forest is fairly good for training even small samples, it fails when there are rare outcomes or rare predictors because the algorithm is based on bootstrap sampling. In Decision Tree, continuous variables are implicitly discretized by the splitting process, losing information along the way. That's why, the AUC of ANN, Random Forest, and Decision Tree are very far from BRBES. So, the produced results of BRBES are better than other methods. Besides, the range of confidence interval of BRBES is highest, and the standard error for BRBES is lowest among other methods. So, it is more reliable than other methods. Thus it can be said that BRBES is performing better than other machine learning techniques.

\section{CONCLUSION}

In this paper, a RESTful API-based BRB expert system has been presented for evaluating TIC. This expert system can handle larger rule bases by providing usability, portability and more computational power. To improve a firm's TIC performance, the firm manager can use this system for evaluating and determining a firm's innovation capabilities, and it will have a great impact on reducing overall uncertainty associated with technological innovation. The RESTful API provides an abstraction layer for the users wishing to use the BRB algorithm for their own domain. A comprehensive study with performance evaluation has also been performed to determine how BRB expert system is performing compared to other machine learning approaches. However, the dataset used for this research is not quite large. So, more data from other firms can be considered if available and more relevant criteria can be examined for evaluating TIC. The security aspects of the system can also be explored.

\section{REFERENCES}

[1] T. S. Durrani, S. M. Forbes, and C. Broadfoot, "An integrated approach to technology acquisition management," International Journal of Technology Management, vol. 17, no. 6, pp. 597-617, 1999.

[2] C.-h. Wang, I.-y. Lu, and C.-b. Chen, "Evaluating firm technological innovation capability under uncertainty," Technovation, vol. 28 , no. 6 , pp. 349-363, 2008.

[3] M. J. Dziura, "Innovation: sources and strategies," International Journal of Technology Management, vol. 21, no. 5-6, pp. 612-627, 2001.
[4] A. Afuah and A. Afuah, "Innovation management: strategies, implementation and profits," 2003

[5] M. S. Hossain, S. Rahaman, A.-L. Kor, K. Andersson, and C. Pattinson, "A belief rule based expert system for datacenter pue prediction under uncertainty," IEEE Transactions on Sustainable Computing, vol. 2, no. 2, pp. 140-153, 2017.

[6] R. C. Yam, J. C. Guan, K. F. Pun, and E. P. Tang, "An audit of technological innovation capabilities in chinese firms: some empirical findings in beijing, china," Research policy, vol. 33, no. 8, pp. 11231140,2004

[7] I.-Y. Lu, C.-B. Chen, and C.-H. Wang, "Fuzzy multiattribute analysis for evaluating firm technological innovation capability," International Journal of Technology Management, vol. 40, no. 1-3, pp. 114-130, 2007.

[8] F. Kong, Z. Zhang, and Y. Liu, "Study on the evaluation of technological innovation capability under uncertainty," in 2008 4th International Conference on Wireless Communications, Networking and Mobile Computing. IEEE, 2008, pp. 1-4.

[9] Y.-L. Cheng and Y.-H. Lin, "Performance evaluation of technological innovation capabilities in uncertainty," Procedia-Social and Behavioral Sciences, vol. 40, pp. 287-314, 2012.

[10] D. Sumrit and P. Anuntavoranich, "Using dematel method to analyze the causal relations on technological innovation capability evaluation factors in thai technology-based firms," International Transaction Journal of Engineering, Management, \& Applied Sciences \& Technologies, vol. 4, no. 2, pp. 81-103, 2013.

[11] R. Karim, K. Andersson, M. S. Hossain, M. J. Uddin, and M. P. Meah, "A belief rule based expert system to assess clinical bronchopneumonia suspicion," in 2016 Future Technologies Conference (FTC). IEEE, 2016, pp. 655-660.

[12] M. S. Hossain, M. S. Khalid, S. Akter, and S. Dey, "A belief rule-based expert system to diagnose influenza," in 2014 9Th international forum on strategic technology (IFOST). IEEE, 2014, pp. 113-116.

[13] R. Ul Islam, K. Andersson, and M. S. Hossain, "A web based belief rule based expert system to predict flood," in Proceedings of the 17th International conference on information integration and web-based applications \& services. ACM, 2015, p. 3.

[14] T. Mahmud, K. N. Rahman, and M. S. Hossain, "Evaluation of job offers using the evidential reasoning approach," Global Journal of Computer Science and Technology, 2013.

[15] T. Mahmud and M. S. Hossain, "An evidential reasoning-based decision support system to support house hunting," International Journal of Computer Applications, vol. 57, no. 21, pp. 51-58, 2012.

[16] M. S. Hossain, S. Rahaman, R. Mustafa, and K. Andersson, "A belief rule-based expert system to assess suspicion of acute coronary syndrome (acs) under uncertainty," Soft Computing, vol. 22, no. 22, pp. 7571-7586, 2018.

[17] M. S. Hossain, F. Ahmed, K. Andersson et al., "A belief rule based expert system to assess tuberculosis under uncertainty," Journal of medical systems, vol. 41, no. 3, p. 43, 2017.

[18] M. S. Hossain, P.-O. Zander, M. S. Kamal, and L. Chowdhury, "Beliefrule-based expert systems for evaluation of e-government: a case study," Expert Systems, vol. 32, no. 5, pp. 563-577, 2015.

[19] M. S. Hossain, I. B. Habib, and K. Andersson, "A belief rule based expert system to diagnose dengue fever under uncertainty," in 2017 Computing Conference. IEEE, 2017, pp. 179-186.

[20] M. R. Sabbir Hridoy, M. S. Hossain, R. U. Islam, and K. Andersson, "A web based belief rule based expert system for assessing flood risk," in Proceedings of the 19th International Conference on Information Integration and Web-based Applications \& Services. ACM, 2017, pp. 434-440.

[21] J. T. Roscoe, Fundamental research statistics for the behavioral sciences [by] John T. Roscoe, 1975. 